\title{
Effectiveness of partial COVID-19 vaccination on the outcome of hospitalized COVID-19 patients during the second pandemic In India
}

Sajal De ( $\nabla$ sajalde@yahoo.com )

All India Institute of Medical Sciences https://orcid.org/0000-0002-8349-125X

Dibakar Sahu

All India Institute of Medical Sciences

Diksha Mahilang

All India Institute of Medical Sciences

Kalleshwara IT

All India Institute of Medical Sciences

Ranganath Ganga

All India Institute of Medical Sciences

Ajoy Behera

All India Institute of Medical Sciences

\section{Brief Communication}

Keywords: COVID-19, vaccination, hospitalization, mortality, disease severity

Posted Date: October 25th, 2021

DOl: https://doi.org/10.21203/rs.3.rs-964720/v1

License: (9) (i) This work is licensed under a Creative Commons Attribution 4.0 International License. Read Full License 


\section{Abstract \\ Background}

This study investigated the effects of COVID-19 vaccination on the outcome of hospitalized COVID-19 patients.

\section{Methods}

We included COVID-19 patients aged $\geq 45$ years and hospitalized between 1st March to 30th June 2021 at our center. We stratified them into unvaccinated and vaccinated (received one or both doses of vaccine).

\section{Results}

Total 1430 COVID-19 patients (male: 876 ) were included. Five hundred thirty-six patients (37.5\%) received a single vaccine dose, and 43 patients (3\%) received two doses before developing COVID-19 disease. The median days of hospital stay of those who survived were ten days (IQR: 8-15). The overall mortality rate, irrespective of vaccination status, was $28.5 \%$. The mortality in the vaccinated was significantly less than the unvaccinated $(25.2 \%$ vs. $30.7 \%, \mathrm{p}<0.05)$. The moderate $(37.3 \%$ vs. $43.4 \%, P<0.01)$ and severe $(16.8 \%$ vs. $21.7 \%$ ) COVID-19 cases were significantly less in the vaccinated.

\section{Conclusion}

The present study showed even a single dose of COVID-19 vaccination significantly reduces the severity of the disease, mortality, and length of hospitalization.

\section{Introduction}

The COVID-19 vaccination in India was launched on 16th January 2021. The vaccination for adults aged $>60$ years with or without comorbidities and adults aged 45 and 60 years with specified comorbidities was rolled out from 1st March 2021. Two vaccines, i.e., Covishield® and Covaxin ${ }^{\circledR}$ were only available during this period. Covaxin $\AA$ is a whole-virion inactivated Vero cell vaccine. Covishield ${ }^{\circledR}$, i.e., ChAdOx1 nCoV-19, a recombinant, replication-deficient chimpanzee adenovirus vector encoding the SARS-CoV-2 Spike (S) glycoprotein. A previous study showed a single dose of Covishield® significantly reduces the incidence of symptomatic COVID-19 and protects against severe disease in individuals aged $\geq 70$ years. ${ }^{1}$ However, the effects of partial COVID-19 immunization on the survival of hospitalized patients are not much known. From the end of March 2021, a sudden surge of COVID-19 cases (second pandemic) was started across different parts of India. This second pandemic in India was probably due to a highly 
infectious B.1.617.2 (delta) variant. ${ }^{2}$ The effectiveness of the above vaccines against delta mutants was also not never investigated during the vaccine development. Two doses of Covishield $\AA$ are less effective in the delta variant than the alpha variant. ${ }^{3}$ Data on the effectiveness of the incomplete vaccination on the outcome of COVID-19 patients are lacking. We retrospectively investigated the effectiveness of COVID-19 vaccination on the outcomes (deaths, duration of hospitalization) and severity of the illness in hospitalized COVID-19 patients.

\section{Patients \& Methods}

We retrospectively collected the data of laboratory-confirmed COVID 19 cases (either real-time polymerase chain reaction or Rapid antigen test for SARS-CoV-2 positive). COVID-19 patients aged 45 years or older and were hospitalized at a tertiary care teaching hospital (Raipur, India) from 1st March 2021 to 30th June 2021 were included. The Intuitional Ethics Committee approved the study protocol

The enrolled subjects were stratified into unvaccinated and vaccinated (received either one or both vaccine doses). We classified the severity of COVID-19 illness into mild, moderate, and severe based on the requirement of oxygen delivery devices and ventilatory support during hospitalization. Mild COVID-19 was those who were either asymptomatic or received supplemental oxygen by a low-flow system (i.e., nasal cannula, face mask). Moderate COVID-19 cases were those who required supplemental oxygen through a high-flow system (non-rebreathing face mask, high flow nasal cannula) for $>24$ hrs duration and ventilator (non-invasive or invasive) support (if any) for $<24 \mathrm{hrs}$. Severe or critically ill COVID-19 was defined as those who required ventilator support (non-invasive or invasive) for $>24$ hours. We collected the vaccination details by telephonic calls to patients or their family members. Univariate and multivariate logistic regression analysis was used to calculate the odds ratio of deaths with a $95 \%$ confidence interval $(\mathrm{Cl})$.

\section{Results}

Among COVID-19 patients admitted during the study period, 1560 (male: 932) met our inclusion criteria. We were unable to collect the vaccination details of 140 patients, and they were excluded from the analysis. The final cohort size was 1430 (male: 854 ) patients of age $59.5 \pm 10.5$ years. Among them, 817 (male: 475 ) were unvaccinated (58.7\%), and 576 (male: 379 ) were vaccinated (41.3\%) at the time of developing COVID-19 infection. The majority of vaccinated (89\%) received Covishield®, as it was only available in the state during the initial days of vaccination. Among the vaccinated, only 43 received fully vaccinated (received two doses of vaccine) before acquiring COVID-19 infection. Eighteen patients were unable to recall their vaccination date. The median interval between the last vaccination dose and microbiologically confirmation for COVID-19 disease was 16 days (IQR: 11-26, range: 0-93).

Total 948 (66.3\%) patients mentioned self-reported comorbidities at the time of hospitalization. Hypertension was the commonest comorbidity (47.3\%), followed by diabetes mellitus (37.8\%). The prevalence of comorbidities between the vaccinated (68.6\%) and unvaccinated $(64.7 \%)$ was similar $(p=$ 
0.07). The overall prevalence of mild, moderate, and severe COVID- 19 was $56.1 \%, 24.2 \%$, and $19.7 \%$, respectively. The mild COVID-19 diseases were significantly higher in the vaccinated $(62.5 \%$ vs. $51.7 \%$, $p<0.001)$. The prevalence of moderate $(20.9 \%$ vs. $26.4 \%, p<0.001)$ and severe $(16.6 \%$ vs. $21.9 \%, p<0.001)$ COVID-19 was significantly less in the vaccinated. The median hospitalization for those who were discharged from the hospital was ten days (IQR: 8-15). The median duration of hospitalization in the vaccinated group was significantly less (median: 10 days; IQR: 7-13; $p<0.01$ ) than the unvaccinated (Median; 11 days, IQR: 8-15).

Total 389 (27.9\%) patients died during hospitalization. In addition, 17 patients died within 15 days after discharge. Therefore, we considered total deaths in our study as 407 patients (28.5\%). The number of deaths in men was significantly more than in women (32.8\% vs. $23.2 \% ; p<0.001)$. Irrespective of vaccination history, deaths recorded in mild, moderate, and severe COVID-19 were $7.5 \%, 37.3 \%, 77.3 \%$, respectively. The mortality in the vaccinated was significantly less than the unvaccinated $(25.2 \%$ vs. $30.7 \%, p<0.05$ ). The unadjusted odds for deaths among the vaccinated was 0.76 ( $95 \% \mathrm{Cl}: 0.60-0.97$, $\mathrm{p}<0.01$ ). The adjusted (adjusting gender, age, and presence of comorbidity) odds for deaths in the vaccinated was 0.67 (95\% Cl: 0.53-0.86, $\mathrm{p}<0.01$ ). The odds for death in those who developed COVID-19 infections within $\leq 20$ days of vaccination was 1.06 (95\% Cl: $0.72-1.58, p>0.05)$

\section{Discussion}

This study provides real-world evidence for the effectiveness of partial COVID-19 on mortality and severity of the disease among hospitalized patients.

A previous study demonstrated that two doses of Covishield $\circledast$ have nearly double effectiveness compared to a single dose. ${ }^{1}$ The effectiveness of Covisheild for the prevention of hospitalization and death starts in the above study observed 14 to 20 days after vaccination. The effectiveness increased up to $60 \%$ during 28 to 34 days after vaccination. On the contrary, we observed those who developed infection $>20$ days of receiving the vaccine were not at lower risk of death. A previous study reported higher odds of a positive COVID-19 report within the initial few days of vaccination. ${ }^{3}$ We observed $25.3 \%$ of our study population developed the infection within ten days of vaccination.

Fewer in our study received Covaxin ${ }^{\circledR}$. Therefore, we weren't able to compare the effectiveness of the two vaccines. The major limitations of the present study were the small cohort and a single-center study.

Our preliminary study showed that even a single dose of vaccination (partial vaccination) reduces deaths, duration of hospitalization, and disease severity. Additional studies are required to evaluate the effectiveness of full immunization in hospitalized COVID-19 patients.

\section{Declarations}

\section{Conflict of Interest}


All authors have no conflicts of interest in this work.

\section{Authors' contributions}

Dr De had full access to all of the data in the study and took responsibility for the data's integrity and the accuracy of the data analysis.

Concept, design, data analysis: Dr. De

Data acquisition: All authors.

Drafting of the manuscript: All authors.

\section{References}

1. Lopez Bernal J, Andrews N, Gower C, Robertson C, Stowe J, et al. effectiveness of the PfizerBioNTech and Oxford-AstraZeneca vaccines on covid-19 related symptoms, hospital admissions, and mortality in older adults in England: test negative case-control study. BMJ. 2021;373:n1088

2. Chakraborty C, Sharma AR, Bhattacharya M, Agoramoorthy G, Lee SS. The current second wave and COVID-19 vaccination status in India. Brain Behav Immun. 2021:S0889-1591(21)00199-9.

3. Lopez Bernal J, Andrews N, Gower C, Gallagher E, Simmons R, et al. Effectiveness of Covid-19 Vaccines against the B.1.617.2 (Delta) Variant. N Engl J Med. 2021;385:585-594. 